\title{
OVERVIEW OF MISMATCH REPAIR PATHWAY
}

\author{
Ananthaneni Anuradha1, Pratibha Ramani², Guduru Vijay Srinivas ${ }^{3}$, Undavalli Suresh Babu ${ }^{4}$, H. K. Puneeth ${ }^{5}$
}

1Phd. Student, Department of Oral Pathology, Saveetha Dental College, Chennai, Tamilnadu, India.

2Professor and HOD, Department of Oral Pathology, Saveetha Dental College, Chennai, Tamilnadu, India.

3 Professos and HOD, Department of Oral Pathology, St. Joseph Dental College, Eluru, Andhra Pradesh, India.

${ }_{4}^{4}$ Associate Professor, Department of ENT, Pinnamaneni Siddhartha Institute of Medical Sciences, Gannavaram Mandal,

Andhra Pradesh, India.

${ }^{5}$ Reader, Department of Oral Pathology, St. Joseph Dental College, Eluru, Andhra Pradesh, India.

\begin{abstract}
BACKGROUND
ABSTRACT

DNA mismatch repair (MMR) has an exclusive chapter in maintaining DNA replication fidelity, mutation avoidance and genome stability. At mechanistic level MMR is quite complex in eukaryotes than prokaryotes. In recent years, many MMR-deficient human cancers specifically hereditary nonpolyposis colorectal cancer (HNPCC), also known as Lynch syndrome (LS), and associated endometrial cancer are allied to mutations in MMR genes. Most of the proteins involved in this pathway are predictably tumour suppressors and defects in the MMR genes convene a strong predisposition for cancer. This review summarizes the literature on MMR, emphasising the role of different MMR proteins in the repair pathway.
\end{abstract}

\section{KEY WORDS}

Mismatch, MSH2, PMS2, Repair.

HOW TO CITE THIS ARTICLE: Anuradha A, Ramani P, Srinivas GV, et al. Overview of mismatch repair pathway. J. Evolution Med. Dent. Sci. 2018;7(51):5479-5483, DOI: 10.14260/jemds/2018/1212

\section{BACKGROUND}

DNA mismatch repair pathway plays a pivotal role in maintaining the genomic integrity during DNA replication, repair and recombination by preventing DNA base mismatches, insertions or deletions. The integrity of the genomic structure is contingent on the precision with which DNA replication is carried out and post replication check out or surveillance. DNA polymerases proofread the replicated DNA and errors are corrected, tailed subsequently by quality control via DNA mismatch repair (MMR) pathway which restitutes the errors that had dodged the DNA polymerase proofreading. MMR makes out mispaired bases, chemically modified bases and insertion-deletion loops that arise during DNA replication and initiates DNA damage response pathway thus reducing the number of replication-associated errors and prevents mutations from becoming permanent in dividing cells. Shortcomings in MMR increase the rate of spontaneous mutations and microsatellite instability (MSI) resulting in hereditary and sporadic cancers in human cells. MSI is due to insertion-deletion loops subsequent to gain or loss of short repeat units within microsatellite sequence.

\section{Mismatch Repair Proteins}

The basic MMR machinery involves two families of proteins MutS and MutL convoluted to form heterodimers in contrast to bacteria, which function as homodimers. Several phases of human MMR pathway are analogous to that in bacteria. Voluminous studies have been done in bacteria with still enigmatic areas in human MMR repair mechanism which is much more complex.

'Financial or Other Competing Interest': None.

Submission 23-11-2018, Peer Review 05-12-2018,

Acceptance 08-12-2018, Published 17-12-2018.

Corresponding Author:

Ananthaneni Anuradha,

Saveetha Dental College,

Eluru, Andhra Pradesh, India.

E-mail: anuradhaundavalli@yahoo.com

DOI: $10.14260 /$ jemds/2018/1212

(c) (1) $(9)$
The elementary method of operation is an excision-repair in which the recently synthesized strand is inspected for discrepancies and the section of the strand containing the inappropriate base is excised and resynthesized, expending a number of suitable DNA repair proteins.(1)

Six MutS homologs are well-known in eukaryotes and these form three MutS complexes - MutS $\alpha$, MutS $\beta$, and MutS $\gamma$. MutS $\alpha$ is a heterodimer of MSH2-MSH6 (also known as G:Tbinding protein, GTBP); MutS $\beta$ is a heterodimer of MSH2MSH3 both of which are crucial for mispair recognition and initiation during the initial stages of MMR mechanism. A third MSH complex, MutS $\gamma$ is a heterodimer of MSH4-MSH5. It has an imperative role in meiotic recombination and is not elucidated in any MMR-related function. MLH1, MLH3, PMS1, and PMS2 are the 4 recognized human MutL homologs. MLH1 heterodimerizes with PMS2, PMS1, or MLH3 to form MutLa

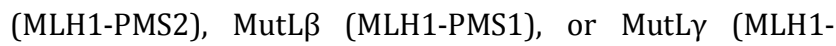
MLH3). Of which homologs MutL $\alpha$ has an obligatory role in MMR whereas MutL $\gamma$ is critical in meiosis, but no specific biological role has been identified for MutL $\beta$ but is reflected as an add-on associate in MMR. Higher levels of MMR proteins are appreciated in multiplying cells than resting cells as replication errors primarily occur in proliferating cells and MMR is framed to correct these.(1),(2)

\section{Mismatch Repair Pathway Recognition of Mismatched DNA}

MutS $\alpha$ makes out base-base mismatches, single base-pair insertion/deletion loops whereas MutS $\beta$ recognizes larger insertion/deletion loops i.e. more than one base-pair but less than 12- base-pair.(3) The crystalline edifice of bacterial DNA has provided an understanding of the mechanism of mispair recognition by MutS. Each monomer of the homodimer contains six structural domains with discrete functions. Domain I corresponds to the mispair recognition by binding to the G: T mismatch and to the unpaired T; by way of only one subunit creating an asymmetry in the dimer. Eukaryotic, MutS is asymmetric because of the presence of heterodimers MSH2-MSH6 and MSH2-MSH3. MSH6 and MSH3 are 
suggested to be the subunits involved in recognition of DNA mismatch. By lacking a large segment of domain, I, MSH4 and MSH5 are meiosis-specific and are not involved in mismatch recognition. The connector domain or the domain II interacts with MutL, domain III typifies the core domain and divides DNA binding and nucleotide binding sites, the periphery of the DNA clamp forms domain IV; domain V encompasses the ATPase domain and dimerization sites; and domain VI or helix-turn-helix domain (HTH) is obligatory for dimerization at moderate protein concentrations. In eukaryotes, similar to MutS, domain V of MSH6 and MSH2 contain ATPase domains that bind ATP with different kinetics and affinities. ${ }^{(4)}$

One stationary model(5) and two moving models i.e. translocation model(6) and the other called as molecular switch or sliding model(7) have been proposed to explain the pathway of communication between DNA and MMR proteins. Recent studies are in favor of the sliding model which proposes that soon after DNA replication the heterodimer of MSH2-MSH6, is tethered to ADP and in an open configuration, scans the newly synthesized DNA for mispairs. Upon encountering a mispair, the heterodimer is anchored by Phe$\mathrm{X}$-Glu to the minor groove of the duplex at the mismatch site to ensign the assembly or nearby localization of the excision repair machinery. When the entire system is cumulated, then the exchange of ADP $\rightarrow$ ATP would be prompted and Phe-X-Glu is released from the site, because of changes in affinity. Then it shifts to a closed, sliding clamp that diffuses along the DNA. The MutS $\alpha$ moving in the $5^{\prime}>3^{\prime}$ directions will eventually signal subsequent repair events. In both models, the role of MutL in downstream signaling and detailing of strand excision is not properly elaborated.(8),(9),(10)

\section{Strand Discrimination}

The intermittent gaps or strand discontinuities in DNA in between the $5^{\prime}$ or $3^{\prime}$ ends of Okazaki fragments on lagging strand of DNA could discriminate parental from daughter strand and persuade the MMR reaction on the nascent strand.(11),(12),(13) PCNA is loaded onto DNA at the pre-existing nick by RFC. PCNA is perhaps a multifunctional matchmaker protein that aids MMR apparatus to distinguish nascent and template strands. Moreover, PCNA intensifies the mismatchbinding specificity of MSH2-MSH6, and also contributes to the conveyance of MSH2-MSH6 onto the mismatched DNA alongside interacting with the mismatch excision enzyme EX01.(14)

\section{Recruitment of Repair Enzymes}

The DNA: MutS $\alpha$ : ATP complex recruits the MutL $\alpha$ to form a ternary complex. This complexing is through its interaction with MutS $\alpha$. MutL $\alpha$ serves as a connecting link between recognition and excision of a mismatch as it activates the endonuclease activity of PMS2. MutL $\alpha$ then diffuses along the DNA until it encounters PCNA that is loaded at a 3' terminus of a nearby nick by RFC. It then displaces the DNA polymerase and proliferating cell nuclear antigen (PCNA) from the nascent daughter strand, and recruits exonucleaseI. The PMS2 subunit of MutL $\alpha$ possesses a cryptic endonuclease activity and brings about additional single strand breaks into the pre-nicked strand as soon as triggered.

\section{Excision of the Mismatch}

The heading of excision in $5^{\prime} \rightarrow 3^{\prime}$ or $3^{\prime} \rightarrow 5^{\prime}$ directions is determined by the position of the nick qualified to the mismatch. Ternary clamps that propagate upstream from 3 $\rightarrow 5^{\prime}$ terminus come across RFC that is tacked at the $5^{\prime}$ terminus of the strand break and in due course displace it by loading EX01. The degradation of the strand in a $5^{\prime} \rightarrow 3^{\prime}$ direction commences once the exonuclease is activated. The single-stranded gap is stabilized by RPA which is a single strand binding protein and is a prerequisite to finish the excision process once the mismatch is removed. At this point, the activity of EXO1 is suppressed by MutLa, thus terminating DNA excision. Ternary Clamps that diffuse downstream 5' $\rightarrow$ 3' terminus come across PCNA molecule that is allied to the $3^{\prime}$ terminus of the strand break. After several events of recruitment and loading, EXO1 is activated resulting in collapse of the region between the preliminary discontinuity and the mismatch. RFC that is allied at the 5' terminus of the break precludes further degradation in the 5 $\rightarrow$ 3' direction i.e. away from the mismatch. DNA bound RPA and MutL $\alpha$ constrain EXO1 activity the minute after the mismatch is removed. An extent of over 1, 000 nucleotides in a single strand of DNA can be excised by EXO1 which is a $5^{\prime} \rightarrow$ 3' exonuclease extending from the nick to the mismatch and terminating about 150 nucleotides past the mismatch.(15)

\section{DNA Resynthesis}

Replicative polymerase Pol $\delta$ is then conscripted by the DNA bound PCNA to the 3' terminus of the preliminary discontinuity. This complex seals the gap and DNA ligase I patches up the remaining nick to complete the restorative process.(16),(17)

\section{Retrospective Analysis of MMR Proteins in Malignancy}

Inactivation of MMR protein function is well known to have numerous biological consequences, including genome instability, resistance to DNA damaging agents including chemotherapeutic drugs,(18) altered class switch recombination and somatic hypermutation of immunoglobulin genes,(19) emergence of pathogenic bacteria, infertility, promotes TNR expansion(20),(21) and increased susceptibility to cancer.(22) Imbalance in the expression of MMR proteins essentially an increased copy number are linked to enhanced mutation rates and development of cancer. Heterocomplexes of MMR proteins, MSH2: MSH6 and MLH1: PMS2 display a pattern of increased expression within the nucleus from $G_{1}$ through $S$ phase and remain relatively high into $\mathrm{G}_{2}$ phase.(23),(24) MSH2 levels increase during the replicative and post-replicative levels of the cell cycle that is at least 12 -fold increase in proliferating cells in contrast to resting cells.(25)

Pereira et al,(26) investigated the expression of MSH2 in head and neck squamous cell carcinoma (HNSCC) and established a down-regulation in $9 \%$ of the samples. Their scrutiny revealed that reduced MSH2 expression in HNSCC tissues was associated with poor overall survival rate and might contribute to high genomic instability resulting in a worse prognosis. Supporting this PMS2-deficient mouse have shown to have decreased MMR efficiency, increased microsatellite instability, as well as an increased susceptibility to develop sarcomas and lymphomas (Baker et al. 1995; Prolla et al. 1998).

Increased expression of MSH2 in tongue squamous cell carcinoma (SCC) was significantly associated with reduced depth of invasion, no evidence of muscular invasion 
prompting the fact that MSH2 increase may be an elicitation of efforts to repair DNA damage in order to impede the advancement of the tumour. But on the contrary, there was also a significant association with shorter disease-free patients and diffuse tumour shape suggesting a highly invasive tumour pattern. Based on these results it was concluded that MSH2 may not be directly related to prognosis but as this protein is related to many other processes, this increase may be linked to their activation. (27)

With the increase in grades of Oral squamous cell carcinoma (OSCC) that is as the tumour becomes less differentiated MSH2 expression is decreased,(28) contrariwise P53 expression increases.(29) Studies have shown that MutS $\alpha$ and MutL $\alpha$ deficient cells are defective in cell cycle arrest in response to DNA damage probably because the cells deficient in MMR fail to phosphorylate p53 and p73 emanating subversive mutations. Majority of erstwhile data suggest that MMR deficiency is associated with increasing grades i.e. more aggressive tumours and poor prognosis. In contrary to this Kato et al., 2015(30), established that MMR deficiency was indicative of a good prognosis in endometrial cancers probably due to sensitivity to adjuvant radiotherapy. Furthermore, high MSH6 was associated with an increased risk of death from primary melanoma.(31) MutS $\alpha$ i.e. MSH6 and MSH2 complex overexpression also obliges as an independent prognostic factor for poor overall survival in patients with OSCC although higher hMSH6 expression was only associated with poor prognosis, whereas MSH2 expression was not (Wagner et al., 2016)(32).

Immunohistochemical staining with MSH2 and MLH1 in samples obtained from low and high risk areas of development of oral cancer in smokers and non-smokers, disclosed normal expression in the basal and suprabasal layers of the squamous epithelium and $\mathrm{MSH} 2$ immunoreactivity was not directly influenced by demographic factors, tobacco smoking or inflammation. (33) Majority of OSCC tissue samples analyzed showed low to no immunohistochemical expression of MLH1 or MSH2 and promoter hypermethylation of MLH1 or MSH2 was detected in $50 \%$ of the DNA.(34) MSH2 with different methylation status in OSCC or head and squamous cell carcinoma was also reported in a number of other studies. (35),(36),(37)

The expression of MMR proteins was not significantly different between benign and malignant salivary gland tumours.(38),(39) The expression of MMR proteins was lower in severe dysplasia. ${ }^{40,41)}$ Moreover, their expression also decreased in potentially malignant disorders like leukoplakia.(42),(43),(28) Lower expression of MMR proteins was evidenced in oral melanomas(44) which also correlated with high aneuploidy ratio.(45) The utility of a two-antibody panel i.e. PMS2 and MSH6 in colorectal carcinoma and extraintestinal tumours such as intestinal carcinomas, endometrial carcinomas, and skin sebaceous neoplasms was reviewed and its applicability was stamped by a loss of expression of MLH1 or PMS2 and MSH2 or MSH6 in almost all the cases.(46) The incidence of cancer was high in germline mutation carriers of DNA mismatch repair genes. Most noteworthy was the involvement of 8 or more organ sites, signifying a need to develop methods to screen for extracolonic cancer also. ${ }^{(47)}$

High microsatellite instability (MSI) was associated with high frequency of absence or abnormal expression of MSH2,
MSH6 and PMS2 proteins in isolation or in combination.(47),(48) Gill et al,(48) also suggested that loss of PMS2 was more likely linked to a germline mutation rather than to the presence of a somatic aetiology. Alternatively, mutations in MLH1 can also secondarily lead to loss of PMS2 expression while retaining MLH1 immunohistochemical expression. High frequency of PMS2 deficiency is evidenced in CRC's when compared to other proteins possibly because (a) in most of the studies the screening was focused on subjects belonging to families with an obvious history of CRC, (b) PMS2 staining was not included in many screening studies based on the unfounded credence of PMS2 having a minor role in MMR, and (c) mutation detection was complicated by the presence of PMS2 pseudogenes. MSH6 mutations are associated with markedly lower cancer risks than MLH1 or MSH2 mutations.(49),(50) The magnitude of colorectal cancer risk in MLH1 and MSH2 mutation carriers was same, whereas MLH1 carriers had a slightly higher risk of endometrial cancer(51),(52) MSH2 deficiency was associated with more extracolonic cancer types like stomach, small bowel, and prostate in comparison to the families with MLH1 or MSH6 defects.

It has been established that colorectal cancers (CRC) tend to arise in field defects that are deficient in DNA repair and deficiencies in expression of PMS2, ERCC1 and XPF proteins are frequently early, and often coordinated with progressive stages of colon cancer.(53) MLH1/MSH2 immunoreactivity in CRC was related to several pathologic features like the tumour site, tumour type, grade of differentiation, nodal status, Crohn's like lymphoid reaction, DNA ploidy pattern and p53 protein expression. Thus MLH1/MSH2 immunoreactivity can be a used as a prognostic evaluator for the management of stage II and III colorectal cancer patients.(54) Reduced MSH2 protein expression has also been shown to be of unfavorable prognostic value in prostate cancer,(55) soft tissue sarcoma, and biliary tract carcinoma.

Lynch syndrome or hereditary nonpolyposis colorectal cancer (CRC) is an inherited autosomal dominant disorder characterized by the development of CRC and other visceral malignancies due to deleterious germline mutations in the DNA mismatch repair (MMR) genes like MLH1, MLH2, MSH6, and PMS2. De Jesus-Monge et al analyzed MLH1 and MSH2 proteins by IHC and determined $4.3 \%$ prevalence of MMR deficiency among 164 Puerto Rican patients with the majority of cases attributable to Lynch syndrome, and this low prevalence rate than other population was attributed to partial MMR protein testing. Lynch syndrome-related CRC was characterized by microsatellite instability and absence of MMR protein expression in the associated tumour.(56),(52) Most of the studies in the literature are pertaining to highrisk population predisposing to CRC and hence Lynch syndrome is underdiagnosed in the general population. Abnormal IHC staining results should be pursued with additional genetic testing irrespective of families meeting the clinical criteria for the diagnosis of Lynch syndrome.(57) Erstwhile data indicate that MMR deficiency is associated with progression of carcinogenesis and more aggressive tumours.

Thus, the cardinal task of MMR is to correct the replication errors and a flawed MMR would marshal elevated spontaneous mutations especially transitions, transversions, and frameshifts. More recently, a surfeit number of cancers 
including colon, endometrium, prostate, head and neck, stomach, ovarian, breast, and pancreatic have been associated with defects in the MMR mechanism. Few of the MMR proteins are also widely-used as a diagnostic biomarker for human cancers. Apart from increased susceptibility to cancer due to genome instability inactivation of MMR proteins are acknowledged to have numerous biological consequences.

\section{Conclusion and Perspectives}

In summary, this review highlights the precise mechanism of MMR and its essential role in upholding genome stability. Diverse proteins work hand in hand in each phase of MMR to sustain the accuracy of DNA. Implication of MMR proteins in a number of tumours reflects the need to elucidate it from a clinical view point.

\section{REFERENCES}

[1] Reyes GX, Schmidt TT, Kolodner RD, et al. New insights into the mechanism of DNA mismatch repair. Chromosoma 2015;124(4):443-62.

[2] Kolodner R. Biochemistry and genetics of eukaryotic mismatch repair. Genes Dev 1996;10(12):1433-42.

[3] Aquilina G, Bignami M. Mismatch repair in correction of replication errors and processing of DNA damage. J Cell Physiol 2001;185(2):145-54.

[4] Warren JJ, Pohlhaus TJ, Changela A, et al. Structure of the human MutSalpha DNA lesion recognition complex. Mol Cell 2007;26(4):579-92.

[5] Guarné A, Ramon-Maiques S, Wolff EM, et al. Structure of the MutL C-terminal domain: a model of intact MutL and its roles in mismatch repair. EMBO J 2004;23(21):4134-45.

[6] Allen DJ, Makhov A, Grilley M, et al. MutS mediates heteroduplex loop formation by a translocation mechanism. EMBO J 1997;16(14):4467-76.

[7] Acharya S, Foster PL, Brooks P, et al. The coordinated functions of the E. coli MutS and MutL proteins in mismatch repair. Mol Cell 2003;12(1):233-46.

[8] Gradia S, Acharya S, Fishel R. The human mismatch recognition complex hmsh2-hmsh6 functions as a novel molecular switch. Cell 1997;91(7):995-1005.

[9] Hsieh P. Molecular mechanisms of DNA mismatch repair. Mutat Res 2001;486(2):71-87.

[10] Stojic L, Brun R, Jiricny J. Mismatch repair and DNA damage signalling. DNA Repair 2004;3(8-9):1091-101.

[11] Fang WH, Modrich P. Human strand-specific mismatch repair occurs by a bidirectional mechanism similar to that of the bacterial reaction. J Biol Chem 1993;268(16):11838-44.

[12] Holmes J, Clark S, Modrich P. Strand-specific mismatch correction in nuclear extracts of human and Drosophila melanogaster cell lines. Proc Natl Acad Sci U S A 1990;87(15):5837-41.

[13] Thomas DC, Roberts JD, Kunkel TA. Heteroduplex repair in extracts of human hela-cells. J Biol Chem 1991;266(6):3744-51.

[14] Bellacosa A. Functional interactions and signaling properties of mammalian DNA mismatch repair proteins. Cell Death Differ 2001;8(11):1076-92.

[15] Jiricny J. The multifaceted mismatch-repair system. Nat Rev Mol Cell Biol 2006;7(5):335-46.
[16] Jascur T, Boland CR. Structure and function of the components of the human DNA mismatch repair system. Int J Cancer 2006;119(9):2030-5.

[17] Chang DK, Ricciardiello L, Goel A, et al. Steady-state regulation of the human DNA mismatch repair system. J Biol Chem 2000;275(24):18424-31.

[18] Li GM. Mechanisms and functions of DNA mismatch repair. Cell Res 2008;18(1):85-98.

[19] Jun SH, Kim TG, Ban C. DNA mismatch repair system. Classical and fresh roles. FEBS J 2006;273(8):1609-19.

[20] Jiricny J. Replication errors: cha(lle)nging the genome. EMBO J 1998;17(22):6427-36

[21] Bak ST, Sakellariou D, Pena-Diaz J. The dual nature of mismatch repair as antimutator and mutator: for better or for worse. Front Genet 2014;5:287.

[22] Buermeyer AB, Deschenes SM, Baker SM, et al. Mammalian DNA mismatch repair. Annu Rev Genet 1999;33:533-64.

[23] Schroering AG, Edelbrock MA, Richards TJ, et al. The cell cycle and DNA mismatch repair. Exp Cell Res 2007;313(2):292-304.

[24] Edelbrock MA, Kaliyaperumal S, Williams KJ. DNA mismatch repair efficiency and fidelity are elevated during DNA synthesis in human cells. Mutat Res 2009;662(1-2):59-66.

[25] Marra G, Chang CL, Laghi LA, et al. Expression of human MutS homolog 2 (hMSH2) protein in resting and proliferating cells. Oncogene 1996;13(10):218996.

[26] Pereira CS, Oliveira M, Barros Lo, et al. Low expression of MSH2 DNA repair protein is associated with poor prognosis in head and neck squamous cell carcinoma. J Appl Oral Sci 2013;21(5):416-21.

[27] Theocharis S, Klijanienko J, Giaginis C, et al. Expression of DNA repair proteins, MSH2, MLH1 and MGMT in mobile tongue squamous cell carcinoma: associations with clinicopathological parameters and patients' survival. J Oral Pathol Med 2011;40(3):218-26.

[28] Jessri M, Dalley AJ, Farah CS. MutS $\alpha$ and MutL $\alpha$ immunoexpression analysis in diagnostic grading of oral epithelial dysplasia and squamous cell carcinoma. Oral Surg Oral Med Oral Pathol Oral Radiol 2015;119(1):74-82.

[29] Helal Tel A, Fadel MT, El-Thobbani AK, et al. Immunoexpression of p53 and hMSH2 in oral squamous cell carcinoma and oral dysplastic lesions in Yemen: relationship to oral risk habits and prognostic factors. Oral Oncol 2012;48(2):120-4.

[30] Kato M, Takano M, Miyamoto M, et al. DNA mismatch repair-related protein loss as a prognostic factor in endometrial cancers. J Gynecol Oncol 2015;26(1):405 .

[31] Alvino E, Passarelli F, Cannavò E, et al. High expression of the mismatch repair protein MSH6 is associated with poor patient survival in melanoma. Am J Clin Pathol 2014;142(1):121-32.

[32] Wagner VP, Webber LP, Salvadori G, et al. Overexpression of MutS $\alpha$ complex proteins predicts poor prognosis in oral squamous cell carcinoma. Medicine (Baltimore) 2016;95(22):e3725. 
[33] Fernandes AM, De Souza VR, Springer CR, et al. Tobacco and inflammation effects in immunoexpression of hMSH2 and hMLH1 in epithelium of oral mucosa. Anticancer Res 2007;27(4B):2433-7.

[34] Czerninski R, Krichevsky S, Ashhab Y, et al. Promoter hypermethylation of mismatch repair genes, hMLH1 and hMSH2 in oral squamous cell carcinoma. Oral Dis 2009;15(3):206-13.

[35] Demokan S, Suoglu Y, Demir D, et al. Microsatellite instability and methylation of the DNA mismatch repair genes in head and neck cancer. Ann Oncol 2006;17(6):995-9.

[36] Sengupta S, Chakrabarti S, Roy A, et al. Inactivation of human MutL homolog 1 and MutS homolog 2 genes in head and neck squamous cell carcinoma tumours and leukoplakia samples by promoter hypermethylation and its relation with microsatellite instability phenotype. Cancer 2007;109(4):703-12.

[37] Wang Y, Irish J, MacMillan C, et al. High frequency of microsatellite instability in young patients with headand-neck squamous-cell carcinoma: lack of involvement of the mismatch repair genes hMLH1 and hMSH2. Int J Cancer 2001;93(3):353-60.

[38] Castrilli G, Fabiano A, La Torre G, et al. Expression of hMSH2 and hMLH1 proteins of the human DNA mismatch repair system in salivary gland tumours. J Oral Pathol Med 2002;31(4):234-8.

[39] Ohki K, Kumamoto H, Ichinohasama R, et al. Genetic analysis of DNA microsatellite loci in salivary gland tumours: comparison with immunohistochemical detection of hMSH2 and p53 proteins. Int J Oral Maxillofac Surg 2001;30(6):538-44.

[40] de Oliveira DH, de Sousa Lopes ML, de Santana Sarmento DJ, et al. Relationship between the epithelial expression of hMLH1, MDM2, and p63 and lower lip carcinogenesis. J Oral Pathol Med 2014;43(5):357-63.

[41] Sarmento DJ, de Almeida WL, Miguel MC, et al. Immunohistochemical analysis of mismatch proteins in carcinogenesis of the lower lip. Histopathology 2013;63(3):371-7.

[42] Caldeira PC, Aguiar MC, Mesquita RA, et al. Oral leukoplakias with different degrees of dysplasia: comparative study of hMLH1, p53, and AgNOR. J Oral Pathol Med 2011;40(4):305-11.

[43] Caldeira PC, Abreu MH, Batista AC, et al. hMLH1 immunoexpression is related to the degree of epithelial dysplasia in oral leukoplakia. J Oral Pathol Med 2011;40(2):153-9.

[44] Lo Muzio L, Nocini P, Mignogna MD, et al. Immunocytochemical detection of hMSH2 and hMLH1 expression in oral melanoma. Anticancer Res 2000;20(2A):741-8
[45] Korabiowska M, Brinck U, Ruschenburg I, et al. Loss of DNA-mismatch repair gene expression in oral melanomas. Oncol Rep 1999;6(4):921-3.

[46] Mojtahed A, Schrijver I, Ford JM, et al. A two-antibody mismatch repair protein immunohistochemistry screening approach for colorectal carcinomas, skin sebaceous tumours, and gynecologic tract carcinomas. Mod Pathol 2011;24(7):1004-14.

[47] Aarnio M, Sankila R, Pukkala E, et al. Cancer risk in mutation carriers of DNA-mismatch-repair genes. Int J Cancer 1999;81(2):214-8.

[48] Gill S, Lindor NM, Burgart LJ, et al. Isolated loss of PMS2 expression in colorectal cancers: frequency, patient age, and familial aggregation. Clin Cancer Res 2005;11(18):6466-71.

[49] Truninger $\mathrm{K}$, Menigatti $\mathrm{M}, \mathrm{Luz} J$, et al. Immunohistochemical analysis reveals high frequency of PMS2 defects in colorectal cancer. Gastroenterology 2005;128(5):1160-71.

[50] Nakagawa H, Lockman JC, Frankel WL, et al. Mismatch repair gene PMS2: disease-causing germline mutations are frequent in patients whose tumours stain negative for PMS2 protein, but paralogous genes obscure mutation detection and interpretation. Cancer Res 2004;64(14):4721-7.

[51] Bonadona V, Bonaiti B, Olschwang S, et al. Cancer risks associated with germline mutations in MLH1, MSH2, and MSH6 genes in lynch syndrome. JAMA 2011;305(22):2304-10.

[52] Zeinalian M, Emami MH, Naimi $A$, et al. Immunohistochemical analysis of mismatch repair proteins in Iranian colorectal cancer patients at risk for lynch syndrome. Iran J Cancer Prev 2015;8(1)11-7.

[53] Facista A, Nguyen H, Lewis C, et al. Deficient expression of DNA repair enzymes in early progression to sporadic colon cancer. Genome Integr 2012;3(1):3.

[54] Lanza G, Gafà R, Santini A, et al. Immunohistochemical test for MLH1 and MSH2 expression predicts clinical outcome in stage II and III colorectal cancer patients. J Clin Oncol 2006;24(15):2359-67.

[55] Tomaszewska R, Okoń K, Stachura J. Expression of the DNA mismatch repair proteins (hMLH1 and hMSH2) in infiltrating pancreatic cancer and its relation to some phenotypic features. Pol J Pathol 2003;54(1):31-7.

[56] Kheirelseid EA, Miller N, Chang KH, et al. Mismatch repair protein expression in colorectal cancer. I Gastrointest Oncol 2013;4(4):397-408.

[57] South CD, Yearsley M, Martin E, et al. Immunohistochemistry staining for the mismatch repair proteins in the clinical care of patients with colorectal cancer. Genet Med 2009;11(11):812-7. 